Pacific Journal of Mathematics

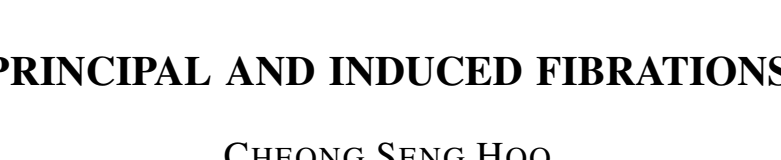




\title{
PRINCIPAL AND INDUCED FIBRATIONS
}

\author{
C. S. Hoo
}

In this paper, the following is proved.

THEOREM. Let $F \stackrel{i}{\rightarrow} E \stackrel{p}{\rightarrow} B$ be a fibration in which $E$ and $B$ have the homotopy type of $C W$ complexes. Suppose that $F$ is $(n-1)$ connected and $B$ is $(m-1)$ connected, where $m, n \geqq 2$. Let $l=\min (m, n), k=\min (2 m-1,2 n)$. Suppose that there exists a map $E \times F \rightarrow E$ of type $(1, i)$. If $\pi_{q}(B)=0$ for all $q \geqq n+l$, then the fibration is Ganea principal. If further $\pi_{q}\left(F^{\prime}\right)=0$ for all $q \geqq n+k$, then the fibration is induced by some map $f: B \rightarrow Y$ for some space $Y$. The dual is also true.

1. All spaces in this paper are provided with a base point, and all maps and homotopies are assumed to preserve base points. In [2], Ganea proved the following.

THEOREM 1. teL $F \stackrel{i}{\rightarrow} E \stackrel{p}{\rightarrow} B$ be a fibration in which $E$ and $B$ have the homotopy type of $C W$ complexes. Suppose that $B$ is $(m-1)$ connected and $F$ is $(n-1)$ connected, where $m, n \geqq 2$. Let $l=$ $\min (m, n)$. Suppose that $i$ maps $\Omega F$ into the centre of $\Omega E$. If $\pi_{q}(B)=0$ for all $q \geqq n+l$ and $\pi_{q}(F)=0$ for all $q \geqq n+2 l-1$, then the fibration is principal and induced by some map $f: B \rightarrow Y$.

In [2], Ganea calls a fibration $F \stackrel{i}{\rightarrow} E \stackrel{p}{\rightarrow} B$ principal if there exists a map $\phi: E \times F \rightarrow E$ and an $H$-structure $m: F \times F \rightarrow F$ such that $\phi(i \times 1)=i m$ and $p \phi=P$ where $P: E \times F \rightarrow B$ is defined by $P(x, y)=$ $p(x)$. It is said to be induced by a map $f: B \rightarrow Y$ for some space $Y$ such that $F \cong \Omega Y$ if it is equivalent to the pull back $\Omega Y \rightarrow W \stackrel{\pi}{\rightarrow} B$ by $f$ of the path space fibration $\Omega Y \rightarrow P Y \rightarrow Y$, that is, if there exists a homotopy equivalence $g: E \rightarrow W$ such that $\pi g=p$. In the rest of the paper, we shall refer to a fibration which is principal in the sense of Ganea as being Ganea-principal.

Various other people have considered principal fibrations slightly differently. In particular, Meyer [4], Porter [6], [7] and Nowlan [5] have considered these questions from various other points of view and have obtained interesting results. In $\S 3$, we shall briefly indicate the connection between their work and our results.

In [2], Ganea says that a map $f: A \rightarrow X$ maps $\Omega A$ into the centre of $\Omega X$ if $(\Omega f)_{\sharp}:[Z, \Omega A] \rightarrow[Z, \Omega X]$ has image contained in the centre of $[Z, \Omega X]$ for all spaces $Z$. It is proved there that this is equivalent 
to the following. Let $X b A \stackrel{L}{\rightarrow} X \vee A$ be the fibre of the usual inclusion $X \vee A \rightarrow X \times A$. Then $f$ maps $\Omega A$ into the centre of $\Omega X$ if and only if $\nabla(1 \vee f) L \cong *: X b A \rightarrow X$ where $\nabla: X \vee X \rightarrow X$ is the folding map. Examples are given in [2] to show that the dimensions imposed on the homotopy of $B$ and $F$ are best possible.

The question of whether or not a given fibration is induced is equivalent to the question of whether or not a map is homotopic to the inclusion of the fibre of some fibration. Thus a fibration $F \rightarrow E \rightarrow B$ is induced means that we can fit it into a sequence $F \rightarrow E \rightarrow B \rightarrow Y$ where any two consecutive maps form a fibre triple. Obviously, a necessary condition is that $F \cong \Omega Y$. Another necessary condition is that $F \rightarrow E$ must be homotopic to the "boundary" map in the Puppe sequence of $E \rightarrow B \rightarrow Y$. Since this may be taken to be $\rho / \Omega Y$ where $\rho: E \times \Omega Y \rightarrow E$ is the operation of the loop space of the base space on the fibre $E$, it follows that $\rho$ is a map of type $(1, \partial)$ where $\partial$ is the "boundary". We make the following definition.

Definition. Let $f: A \rightarrow X$ be a map. We say that $f$ is cyclic if $\nabla(1 \vee f): X \vee A \rightarrow X$ extends to $X \times A$, that is, if there exists a map $\phi: X \times A \rightarrow X$ of type $(1, f)$.

The property of being cyclic is a property of the homotopy class of $f$. We observe that if $F \stackrel{i}{\rightarrow} E \stackrel{p}{\rightarrow} B$ is induced by some map $f: B \rightarrow$ $Y$, then $F \cong \Omega Y$, and $i$ may be taken to be the boundary $\partial$ in the Puppe sequence of $E \stackrel{p}{\rightarrow} B \stackrel{f}{\rightarrow} Y$. Hence $i$ is cyclic.

We note that if $f: A \rightarrow X$ is cyclic, then $f$ maps $\Omega A$ into the centre of $\Omega X$. This follows from the fibration $X b A \rightarrow X \vee A \rightarrow X \times$ $A$. If $\nabla(1 \vee f): X \vee A \rightarrow X$ extends to $X \times A$, then clearly $\nabla(1 \vee f) L \cong *$. We intend to replace the condition " $i$ maps $\Omega F$ into the centre of $\Omega E$ " in Theorem 1 by the stronger condition " $i$ is cyclic." This is intended to enable us to deduce a stronger conclusion. However, we observe that, under the conditions of Theorem 1, the two statements are equivalent. This follows from the following.

THEOREM 2. Let $f: A \rightarrow X$ be a map and suppose that $A$ is $(m-1)$ coonected and $X$ is $(n-1)$ connected. Let $l=\min (m, n)$. Suppose that $f$ maps $\Omega A$ into the centre of $\Omega X$ and that $\pi_{j}(X)=0$ for all $j \geqq m+n+l-1$. Then $f$ is cyclic.

Proof. Consider the fibration $X b A \stackrel{L}{\rightarrow} X \vee A \rightarrow X \times A$. By hypothesis, $\nabla(1 \vee f) L \cong *$. We may factor the inclusion $X \vee A \rightarrow$ $X \times A$ as 


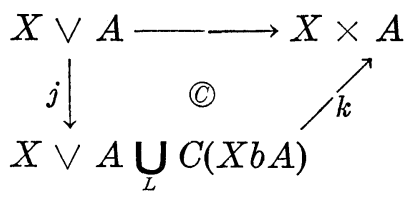

where $k$ extends the inclusion $X \vee A \subset X \times A$ by mapping $C(X b A)$ to the base point. Now from the cofibration $X b A \stackrel{L}{\rightarrow} X \vee A \stackrel{j}{\rightarrow} X \vee A \bigcup_{L}$ $C(X b A)$, since $\nabla(1 \vee f) L \cong{ }^{*}$, we have a map $g: X \vee A \cup_{L} C(X b A) \rightarrow X$ such that $g j \cong \nabla(1 \vee f)$. Now consider the following situation

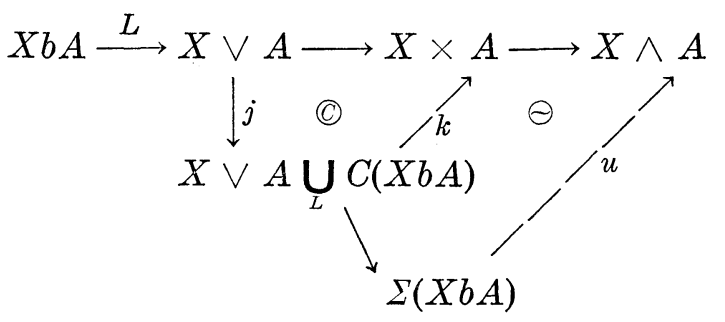

where $X \times A \rightarrow X \wedge A$ is the cofibre of $X \vee A \rightarrow X \times A$ and $X b A \stackrel{L}{\rightarrow}$ $X \vee A \stackrel{j}{\rightarrow} X \vee A \bigcup_{L} C(X b A) \rightarrow \Sigma(X b A) \rightarrow$ is the Puppe sequence of the cofibration, and $u$ is determined in the obvious way. Since $X b A \cong$ $\Sigma(\Omega X \wedge \Omega A)$ (see [1]) it is easily calculated that $X b A$ is $n+m-2$ connected. Hence $X \vee A \rightarrow X \times A$ is $n+m-1$ connected. Also $X \times A$ is $(l-1)$ connected. Applying the Serre theorem, which is dual to the Blakers-Massey theorem (see [3]), we see that $u$ is $n+m+l-1$ connected. Hence by the 5 -lemma, it follows that $k$ is $n+m+l-1$ connected. Since $\pi_{j}(X)=0$ for all $j \geqq n+m+l-1$, by obstruction theory, we can find a map $\phi: X \times A \rightarrow X$ such that $\phi k \cong g$. Hence $\phi k j \cong g j \cong \nabla(1 \vee f)$, where $k j: X \vee A \subset X \times A$ is the inclusion. Hence $f$ is cyclic.

REMARK. Thus in Theorem 1, we may replace the statement " $i$ maps $\Omega F$ into the centre of $\Omega E$ " by " $i$ is cyclic."

We need the following two facts due to Ganea [2]. Suppose $F \stackrel{i}{\rightarrow} E \stackrel{p}{\rightarrow} B$ is a fibration and suppose that $X \stackrel{f}{\rightarrow} Y \stackrel{g}{\rightarrow} Z$ is a triple, that is, $g f=*$. Suppose that have maps $\phi: X \rightarrow F, \varepsilon: Y \rightarrow E$ such that $\varepsilon f \cong i \phi$. Let $h_{t}: X \rightarrow E$ be a homotopy such that $h_{0}=i \phi, h_{1}=$ $\varepsilon f$. Then $\phi, \varepsilon$ and $h_{t}$ define a map $\lambda: Y \bigcup_{f} C X \rightarrow E \bigcup_{i} C F$ by $\lambda(y)=$ $\varepsilon(y)$ and

$$
\lambda(s x)= \begin{cases}2 s \phi(x) & 0 \leqq s \leqq \frac{1}{2} \\ h_{2 s-1}(x) & \frac{1}{2} \leqq s \leqq 1\end{cases}
$$


Let $k: Y \cup C X \rightarrow Z$ extend $g$ by mapping $C X$ to the base point, and let $r: E \cup C F \rightarrow B$ extend $p$ by mapping $C F$ to the base point. Then the triangles in the following diagram commute.

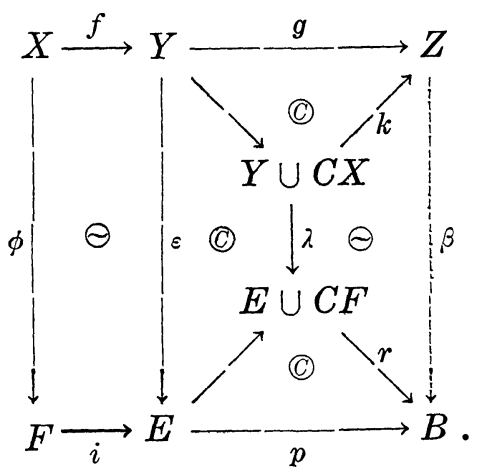

We have the following result.

LEMmA 1 (Ganea [2]). Suppose that in the above situation there exist a map $\beta: Z \rightarrow B$ such that $\beta k \cong r \lambda$. Then we can find maps $\phi_{1} \cong \phi, \varepsilon_{1} \cong \varepsilon$ making the squares in the following diagram commutative.

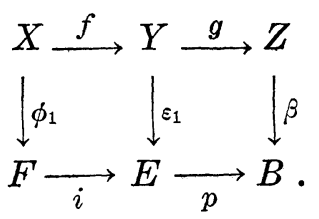

Proof. See Lemma 1.1 of [2].

We also need the following.

Theorem 3 (Ganea [2]). Suppose that $F \stackrel{i}{\rightarrow} E \stackrel{p}{\rightarrow} B$ is a fibration with $E$ and $B$ having the homotopy type of $C W$ complexes. Suppose that $B$ is $(m-1)$ connected and $F$ is $(n-1)$ connected, $m, n \geqq 1$. Suppose that $\pi_{q}(F)=0$ for all $q \geqq n+2 m-1$. If the fibration is Ganea-principal and if there there exists a space $Y$ and a homotopy equivalence $F \rightarrow \Omega Y$ which is also an $H$-map, then the fibration is induced by some map $f: B \rightarrow Y$.

We now state our main result.

THEOREM 4. Let $F \stackrel{i}{\rightarrow} E \stackrel{p}{\rightarrow} B$ be a fibration in which $E$ and $B$ have the homotopy type of $C W$ complexes. Suppose that $F$ is 
$(n-1)$ connected and $B$ is $(m-1)$ connected, $m, n \geqq 2$. Let $l=$ $\min (m, n), k=\min (2 m-1,2 n)$. Suppose that $i$ is cyclic. If $\pi_{q}(B)=0$ for all $q \geqq n+l$, then the fibration is Ganea-principal. Further, if $\pi_{q}(F)=0$ for all $q \geqq n+k$, then the fibration is induced by some map $f: B \rightarrow Y$ for some space $Y$.

Proof. We assume that $\pi_{q}(B)=0$ for all $q \geqq n+l$. Since $i$ is cyclic, we can find a map $\phi: E \times F \rightarrow E$ of type $(1, i)$. We factor

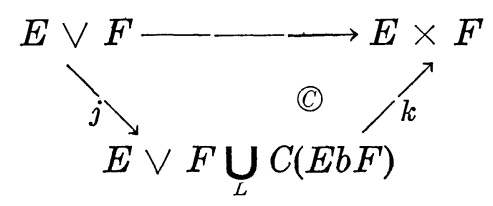

that is, $k j: E \vee F \rightarrow E \times F$ is the usual inclusion. Let $P: E \times F \rightarrow$ $B$ be given by $P(x, y)=p(x)$. Then $P k j=p \dot{\phi} k j$. Now, in the Puppe sequence of the cofibration

$$
E b F \stackrel{L}{\longrightarrow} E \vee F \stackrel{j}{\longrightarrow} E \vee F \cup C(E b F) \longrightarrow \Sigma(E b F) \longrightarrow \cdots
$$

there is an operation

$[E \vee F \cup C(E b F), B] \times[\Sigma(E b F), B] \longrightarrow[E \vee F \cup C(E b F), B]$

and $P k j=p \phi k j$ if and only if we can find a map $\beta: \Sigma(E b F) \rightarrow B$ such that $P k \cong p \phi k T \beta$ rel. $E \vee F$, where we denote the operation by $T$. It is easily calculated that $\Sigma(E b F)$ is $n+l-1$ connected. Since $\pi_{q}(B)=0$ for all $q \geqq n+l$, there is no obstruction to nullhomotopy of $\beta$, that is, $\beta \cong *$. Let $\varepsilon: \Sigma(E b F) \rightarrow E$ be the constant map. Then $\beta \cong p \varepsilon$, that is, $P k \cong p \phi k \top p \varepsilon$ rel. $E \vee F$. Let $g_{0}=$ $\phi k T \varepsilon: E \vee F \cup C(E b F) \rightarrow E$. Then $P k \cong p g_{0}$ rel. $E \vee F$. Note also that $g_{0} j=(\phi k T \varepsilon) j=\phi k j$. Since $P k \cong p g_{0}$ rel. $E \vee F$, and since $p$ is a fibration, we can find a homotopy $g_{t}: E \vee F \cup C(E b F) \rightarrow E$ such that $p g_{1}=P k$, and $p g_{t} d=*$ where $d=j(i \vee 1): F \vee F \rightarrow E \vee F \rightarrow$ $E \vee F \cup C(E b F)$. Hence we can find a homotopy $\nabla_{t}: F \vee F \rightarrow F$ such that $i \nabla_{t}=g_{t} d$. Hence $i \nabla_{0}=g_{0} d=g_{0} j(i \vee 1)=\phi k j(i \vee 1)=\nabla(1 \vee i)(i \vee 1)=$ $i \nabla$, that is, $\nabla_{0}=\nabla$ and $\nabla_{1} \cong \nabla$. We can form the following diagram

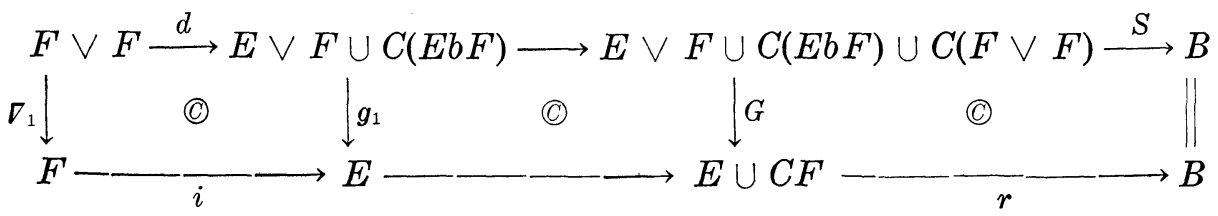

where $G$ is induced by $\nabla_{1}$ and $g_{1}, r$ extends $p: E \rightarrow B$ by mapping $C F$ to the base point, and $S$ extends $P k: E \vee F \cup C(E b F) \rightarrow B$ by mapping $C(F \vee F)$ to the base point. We observe that $g_{1} \cong g_{0} \cong \phi k$. 
Let $H_{t}: E \vee F \cup C\left(E b F^{\prime}\right) \rightarrow E$ be a homotopy such that $H_{0}=g_{1}, H_{1}=$ $\phi k$. Let $h_{t}=H_{t} d: F \vee F \rightarrow E \vee F \cup C(E b F) \rightarrow E$. Then $h_{0}=H_{0} d=$ $g_{1} d=i \nabla_{1}, h_{1}=H_{1} d=\phi k d$. Thus we have the following diagram

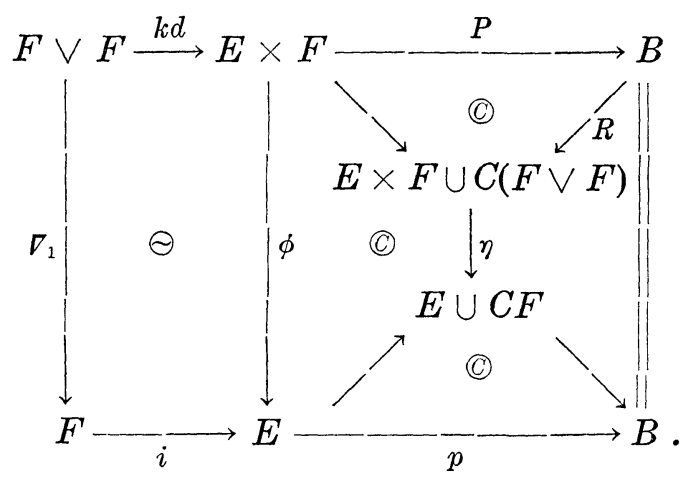

Here $r$ extends $p$ by mapping $C F$ to the base point, and $R$ extends $P$ by mapping $C(F \vee F)$ to the base point. Also the maps $\nabla_{1}, \phi$ and the homotopy $h_{t}$ induce $\eta$. We claim that $r \eta \cong R$. In fact, the map $k: E \vee F \cup C(E b F) \rightarrow E \times F$ gives the following diagram

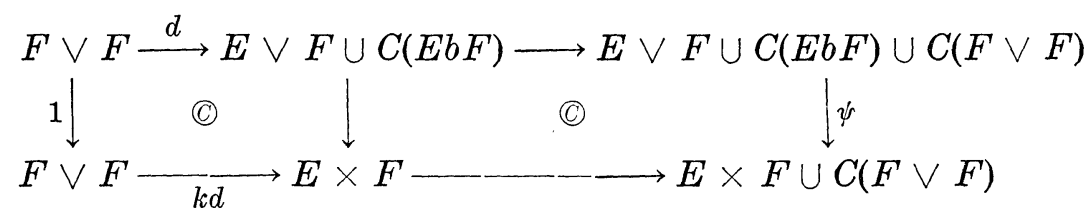

where $\psi$ is induced by $k$ and 1 . Then we check that $\eta \psi \cong G, R \psi=$ $S$. Hence $r \eta \psi \cong r G=S=R \psi$. Since $k$ is $(n+2 l-1)$ connected, by the 5-lemma, it follows that $\psi$ is also $n+2 l-1$ connected. Since $\pi_{q}(B)=0$ for all $q \geqq n+l$, there is no obstruction to a homotopy between $r \eta$ and $R$. Hence $r \eta \cong R$. We now apply Lemma 1 and conclude that we have maps $\nabla_{1}^{\prime}: F \vee F \rightarrow F, \phi^{\prime}: E \times F \rightarrow E$ with $\nabla_{1}^{\prime} \cong$ $\nabla_{1} \cong \nabla$ and $\phi^{\prime} \cong \phi$, and

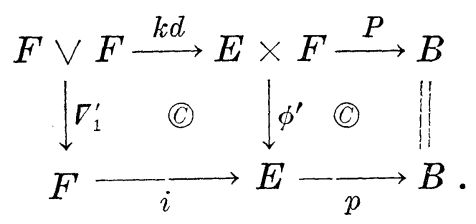

Hence $p \phi^{\prime}(i \times 1)=P(i \times 1)=*$. This means that we can find a map $m: F \times F \rightarrow F$ with $i m=\phi^{\prime}(i \times 1)$. Let $t: F \vee F \rightarrow F \times F$ be the inclusion. Then $i m t=\phi^{\prime}(i \times 1) t=\phi^{\prime} k d$ since $(i \times 1) t=k d$, that is, $i m t=\phi^{\prime} k d=i \nabla_{1}^{\prime}$. Hence $m t=\nabla_{1}^{\prime} \cong \nabla$. Thus $m$ is an $H$-structure. Since $i m=\phi^{\prime}(i \times 1)$ we have the diagram 


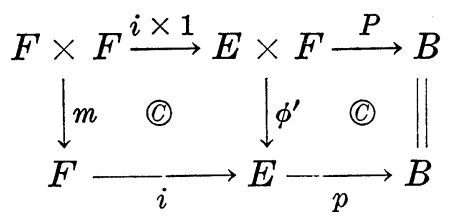

that is, the fibration is Ganea-principal.

Now suppose in addition that $\pi_{q}(F)=0$ for all $q \geqq n+k$. Then $\pi_{q}(F)=0$ for $q \geqq 3 n$. Since $F$ is an $H$-space and is $(n-1)$ connected, it follows by Theorem $\mathrm{C}$ of [3] that there is a homotopy equivalence $\theta: F \rightarrow \Omega Y$ which is also an $H$-map, for some space $Y$. In fact, $Y$ may be constructed as follows. Applying the Hopf construction to the multiplication $m: F \times F \rightarrow F$, we get a map $\Sigma(F \wedge F) \rightarrow \Sigma F$, and hence $\Sigma(F \wedge F) \rightarrow \Sigma F \stackrel{v}{\rightarrow} \Sigma F \cup C \Sigma(F \wedge F)$. Here $\Sigma F \cup C \Sigma(F \wedge F)=$ $F P$ is the $F$-projective plane. Let $(F P)_{3 n}$ be the $3 n$-Postnikov section of $F P$ and let $\pi: F P \rightarrow(F P)_{3 n}$ be the projection. We take $Y=(F P)_{3 n}$ and $\theta$ to be the map $F \stackrel{\bar{v}}{\rightarrow} \Omega(F P) \stackrel{2 \pi}{\longrightarrow} \Omega(F P)_{3 n}, \bar{v}$ being the adjoint of $v$. It is easily seen that $\bar{v}$ is an $H$-map and hence $\theta$ is an $H$-map. The connectivity of $\bar{v}$ may be calculated by the Blakers-Massey theorem, and hence, it may be seen that $\theta$ is a homotopy equivalence. The proof of the theorem is now completed by applying Theorem 3 .

REMARK 1. We observe that we have separated the conditions on $B$ and $F$, and from each, we have deduced a conclusion. Ganea's Theorem 1, above, uses both the conditions on $B$ and $F$ to deduce the conclusion that the fibration is Ganea-principal. Our proof shows that the conclusion that the fibration is Ganea-principal uses only the condition on the homotopy of $B$.

REMARK 2. If $m \leqq n$, our theorem and that of Ganea are the same. However, if $m<n$, our theorem improves that of Ganea by allowing $F$ to have an extra homotopy group. Thus our condition allows the fibration to be Ganea-principal even if $\pi_{3 n-1}(F) \neq 0$, while Ganea's theorem requires that $\pi_{3 n-1}(F)=0$.

REMARK 3. The dimension condition on the homotopy of $B$ is best possible. This is shown by the example given in [2]. Let $Q$ be the rationals and let $n \geqq 4$ be even. Consider the fibration $K(Q, n) b K(Q, n) \rightarrow K(Q, n) \vee K(Q, n) \rightarrow K(Q, n) \times K(Q, n)$. Now $K(Q, n) b K(Q, n) \cong \Sigma(K(Q, n-1) \wedge K(Q, n-1))$. Since $n$ is even, $(n-1)$ is odd, and hence $K(Q, n-1) \cong K^{\prime}(Q, n-1)$. Hence

$$
K(Q, n) b K(Q, n) \cong \Sigma K^{\prime}(Q, 2 n-2)=K^{\prime}(Q, 2 n-1) \cong K(Q, 2 n-1) .
$$

Thus we have a fibration 


$$
K(Q, 2 n-1) \longrightarrow K(Q, n) \vee K(Q, n) \longrightarrow K(Q, n) \times K(Q, n) .
$$

Since the fibre is a single Eilenberg-MacLane complex, by a classical result of Serre, this fibration is induced. It can only be induced by a map $K(Q, n) \times K(Q, n) \rightarrow K(Q, 2 n)$. Thus we have a fibration

$$
K(Q, n) \vee K(Q, n) \longrightarrow K(Q, n) \times K(Q, n) \longrightarrow K(Q, 2 n) \text {. }
$$

Observe that here $\pi_{n+l}(K(Q, 2 n)) \neq 0, n+l$ being $2 n$ here. All the other conditions of the theorem are satisfied. This fibration is not Ganea-principal since $K(Q, n) \vee K(Q, n)$ is not an $H$-space.

REMARK 4. We do not know if the dimension condition on the homotopy of $F$ in Theorem 4 is best possible or not. However, we can say that if it is not best possible, then the best possible is the condition $\pi_{q}(F)=0$ for all $q \geqq n+k+1$. This is because we have the following example. Let $F=K\left(Z_{3}, 3 ; Z_{9}, 10 ; \lambda u(\beta u)^{2}\right)$ be the 2stage Postnikov system, where $u \in H^{3}\left(Z_{3}, 3 ; Z_{3}\right)$ is the fundamental class, $\beta$ is Bockstein operator, and $\lambda$ is induced by the coefficient homomorphism $Z_{3} \subset Z_{9}$. Then $F$ is an $H$-space but not a loop-space (see page 599 of [3]). Thus $F \rightarrow F \rightarrow^{*}$ is not induced. The map $F \rightarrow F$ here is cyclic since an identity map is cyclic if and only if the space is an $H$-space. Thus all the conditions of Theorem 4 are satisfied except that $\pi_{n+k+1}(F) \neq 0$. In fact, we only have $\pi_{q}(F)=0$ for all $q \geqq n+k+2$. Thus the best possible condition on $F$ in Theorem 4 is either $\pi_{q}(F)=0$ for all $q \geqq n+k$ or $\pi_{q}(F)=0$ for all $q \geqq n+$ $k+1$.

Theorem 4 admits the following application. We assume here that all our spaces have the homotopy type of $C W$ complexes. We recall that if we have a fibration $F \stackrel{i}{\rightarrow} E \stackrel{p}{\rightarrow} B$ where $E$ and $B$ are $H$-spaces, then if $p$ is an $H$-map, it follows that $F$ can be given an $H$-structure so that $i$ is an $H$-map. Stasheff in [8] gives a converse under some restrictions.

THEOREM 5 (Stasheff). Let $F \stackrel{i}{\rightarrow} E \stackrel{p}{\rightarrow} B$ be a fibration in which $E$ and $B$ are $H$-spaces. If $F$ has a multiplication, then $p$ is an $H$-map with respect to some multiplication on $E$, provided that $E$ is $(n-1)$ connected, $\pi_{q}(E)=0$ for $q \geqq n+m$, where $m \geqq n+1$, and $B$ is $(m-1)$ connected and $\pi_{q}(B)=0$ for $q \geqq n+m$.

We observe that if $E$ is an $H$-space, then $i$ is automatically cyclic. In fact, if $m: E \times E \rightarrow E$ is the $H$-structure in $E$, then the map $E \times F \rightarrow E$ of type $(1, i)$ required can be taken to be $m(1 \times i)$. Using this fact, it follows from Theorems 4 and 5 that we have the following. 
THEOREM 6. Let $F \stackrel{i}{\rightarrow} E \stackrel{p}{\rightarrow} B$ be a fibration in which $F$ is $(n-1)$ connected, $B$ is $(m-1)$ connected, and $\pi_{q}(B)=0$ for $q \geqq 2 n$, where $m \geqq n+1$. Suppose that $E$ and $B$ are $H$-spaces. Then $p$ is an $H$-map for some multiplication on $E$.

2. These theorems can be dualized. We state the dual of Theorem 4. Recall that a cofibration $A \stackrel{d}{\rightarrow} X \stackrel{f}{\rightarrow} C$ is Ganea-principal if we can find a co-H-structure $m: C \rightarrow C \vee C$ and a map $\phi: X \rightarrow X \vee C$ such that the following diagram commutes

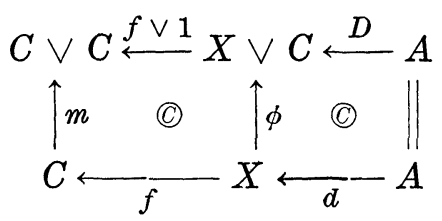

where $D(a)=(d(a), *)$. We are following the terminology of [2]. The cofibration is induced if there is a space $Y$ and a map $g: Y \rightarrow A$ such that the cofibration is equivalent to the cofibration strictly induced by $g$ from $Y \rightarrow C Y \rightarrow \Sigma Y$, that is, to the triple $A \rightarrow A \cup_{g} C Y \rightarrow$ $\Sigma Y$, This means that there is a homotopy-equivalence $X \rightarrow A \mathrm{U}_{g} C Y$ such that

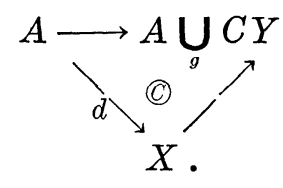

For a 1-connected $C W$ complex $K$, we write $\operatorname{dim} K \leqq n$ to indicate that $H_{n}(K)$ is free and $H_{q}(K)=0$ for $q>n$. We say that a map $f: X \rightarrow A$ is cocyclic if the map $(1 \times f) \Delta: X \rightarrow X \times A$ is compressible into $X \vee A$. This is homotopy property of $f$. The dual of Theorem 4 is the following.

THEOREM 7. Let $A \stackrel{d}{\rightarrow} X \stackrel{f}{\rightarrow} C$ be a cofibration in which $(X, A)$ is a $C W$ pair. Suppose that $A$ is $(m-1)$ connected, and that $C$ is $n$-connected, $m \geqq 2, n \geqq 1$. Suppose that $f$ is cocyclic. If $\operatorname{dim} A \leqq$ $n+\min (m-1, n)$, then the cofibration is Ganea-principal. If further $\operatorname{dim} C \leqq n+\min (2 m-1,2 n)$, then the cofibration is induced.

Proof. Dualize the proof of Theorem 4.

3. We now briefly consider the connection between our results and the work of Meyer [4], Nowlan [5] and Porter [6], [7]. We refer the reader to these papers for detailed definitions, but we shall 
indicate how their notions can be expressed in our terminology. Let $F \stackrel{i}{\rightarrow} E \stackrel{p}{\rightarrow} B$ be a fibration. Then it is an $H$-fibration in the sense of Meyer [4] if it is Ganea-principal, with $i$ being cyclic and $\phi$ being a map of type $(1, i)$. A principal fibration in the sense of Meyer [4] is an induced fibration in the sense of Ganea [2]. All the results in [4] concerning principal fibrations hold for induced fibrations, and all the results concerning $H$-fibrations hold for Ganea-principal fibrations.

An $H$-fibration in the sense of Porter [6] is also Ganea-principal with $i$ being cyclic and $\phi$ being of type $(1, i)$. We might emphasise that our definition of a Ganea-principal fibration does not require $i$ to be cyclic and does not require $\phi$ to be of type $(1, i)$. However, a simple examination shows that most of the results in Meyer [4] on $H$-fibrations and most of those in Porter [6] on $H$-fibrations do not require these extra conditions. They merely require that the fibrations be Ganea-principal. In particular, we mention that Theorem 1 of Porter [6] holds for Ganea-principal fibrations. There is no need to assume that $i$ is cyclic or that $\phi$ is of type $(1, i)$. The proof carries over word for word. Thus we have the following which we shall attribute to Porter.

THEOREM 8 (Porter). Let $F \stackrel{i}{\rightarrow} E \stackrel{p}{\rightarrow} B$ be Ganea-principal, with $B$ being path-connected. Let $g_{1}, g_{2}: X \rightarrow E$ be maps with $p g_{1} \cong p g_{2}, X$ being a $C W$ complex. Then there exists a map $u: X \rightarrow F$ with $\phi\left(g_{2} \times u\right) \Delta \cong g_{1}$, where $\Delta: X \rightarrow X \times X$ is the diagonal map.

In [7] Porter defines his principal fibrations to be Ganea-principal with $(F, m)$ being an associative $H$-space, and the map $\phi: E \times F \rightarrow E$ of type $(1, i)$ is required to be an associative action of $E$. Let $F \stackrel{i}{\rightarrow} E \stackrel{p}{\rightarrow} B$ be such a principal fibration and let $f: B^{\prime} \rightarrow B$ be a map. Let $E_{f}=\left\{(b, e)\right.$ in $\left.B^{\prime} \times E \mid f(b)=p(e)\right\}$. Then $F \rightarrow E_{f} \rightarrow B^{\prime}$ is also a principal fibration and

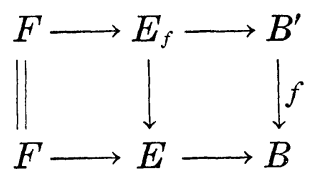

is a homomorphism of principal fibrations. We ask the reader to refer to Porter [7] for the definitions of the various terms we shall be using.

Let $F \stackrel{i}{\rightarrow} E \stackrel{p}{\rightarrow} B$ and $F^{\prime} \stackrel{i^{\prime}}{\rightarrow} E^{\prime} \stackrel{p^{\prime}}{\rightarrow} B^{\prime}$ be fibrations. Then we have the principal fibrations $\Omega B \rightarrow E_{p} \rightarrow E, \Omega B^{\prime} \rightarrow E_{p^{\prime}} \rightarrow E^{\prime}$. Theorem 12 of Porter [7] says that there exists a map $f: B \rightarrow B^{\prime}$ such that $F \rightarrow$ $E \rightarrow B$ is equivalent to the fibration induced by $f$ from $F^{\prime} \rightarrow E^{\prime} \rightarrow B^{\prime}$ 
if and only if there exists a strong homotopy homomorphism of principal fibrations

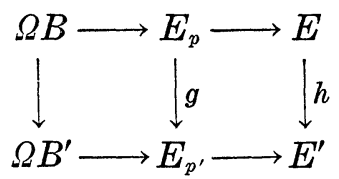

with $g$ being a homotopy equivalence. Our results concern the case where $F^{\prime} \rightarrow E^{\prime} \rightarrow B^{\prime}$ is a path space fibration $\Omega B^{\prime} \rightarrow P B^{\prime} \stackrel{\pi}{\rightarrow} B^{\prime}$. Thus under the conditions stated there on the homotopy of $B$ and $F$, our Theorem 4 says that there exists a strong homotopy homomorphism

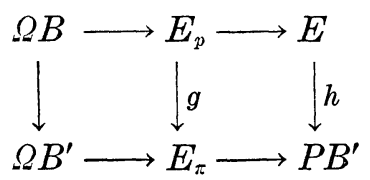

with $g$ being a homotopy equivalence.

Nowlan's $H$-fibrations [5] are our Ganea-principal fibrations. Nowlan considers fibrations in which an associative $H$-space $(F, m)$ operates, but not necessarily associatively. Such fibrations are called $A_{1}$-principal fibre spaces. For example, all the various $H$-fibrations are such fibre spaces, and Ganea-principal fibrations are also such fibre spaces if $(F, m)$ is associative. If instead of requiring that the action be associative, we only require that it be homotopy associative, that is, that the following diagram homotopy commutes

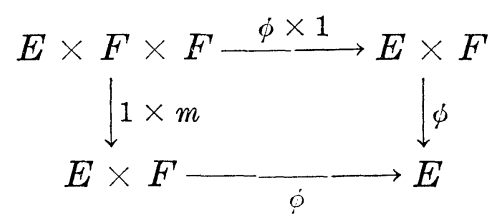

then we get an $A_{2}$-principal fibre space. Thus a principal fibration in the sense of Porter [7] is an $A_{2}$-principal fibre space. An $A_{n}$ principal fibre space is one where the action of $F$ on $E$ satisfies higher homotopy conditions. Nowlan [5] obtains a classification theorem. The notion of an $A_{\infty}$-principal fibre space is also obtained. Nowlan proves the following.

Theorem (Nowlan). $p: E \rightarrow B$ is fibre homotopy equivalent to an induced fibre space if and only if $E$ admits an $A_{\infty}$-action of $\Omega B$.

\section{REFERENCES}

1. T. Ganea, A generalization of the homology and homotopy suspension, Comment. Math. Helv., 39 (1965), 295-322. 
2. T. Ganea, Induced fibrations and cofibrations, Trans. Amer. Math. Soc., 127 (1967), 442-459.

3. P. J. Hilton, Remark on loop spaces, Proc. Amer. Math. Soc., 15 (1964), 596-600.

4. J. P. Meyer, Principal fibrations, Trans. Amer. Mhth. Soc., 107 (1963), 177-185.

5. R. A. Nowlan, $A_{n}$ actions on fibre spaces, Indiana Univ. Math. J., 21 (1971), 285-313.

6. G. J. Porter, H-fibrations, Quart. J. Math., 22 (1971), 23-31.

7. - Homomorphisms of principal fibration: applications to classification, induced fibrations, and the the extension problem, Illinois J. Math., 16 (1972), 41-60.

8. J. Stasheff, Extensions of H-spaces, Trans. Amer. Math. Soc., 105 (1962), 126-135.

Received December 6, 1975. This research was supported by NRC Grant A3026.

UNIVERSITY OF AlBerta

AND

OXFORd University, OXFORD, ENGLAND 


\section{PACIFIC JOURNAL OF MATHEMATICS}

\section{EDITORS}

RICHARD ARENS (Managing Editor)

University of California

Los Angeles, California 90024

\section{J. DugunduI}

Department of Mathematics University of Southern California Los Angeles, California 90007

D. Gilbarg and J. Milgram

Stanford University

Stanford, California 94305

\author{
R. A. Beaumont \\ University of Washington \\ Seattle, Washington 98105
}

\section{ASSOCIATE EDITORS}
B. H. NeUmanN
F. WOLF
K. YosHIDA

\section{SUPPORTING INSTITUTIONS}

\author{
UNIVERSITY OF BRITISH COLUMBIA \\ CALIFORNIA INSTITUTE OF TECHNOLOGY \\ UNIVERSITY OF CALIFORNIA \\ MONTANA STATE UNIVERSITY \\ UNIVERSITY OF NEVADA \\ NEW MEXICO STATE UNIVERSITY \\ OREGON STATE UNIVERSITY \\ UNIVERSITY OF OREGON \\ OSAKA UNIVERSITY
}

\author{
UNIVERSITY OF SOUTHERN CALIFORNIA \\ STANFORD UNIVERSITY \\ UNIVERSITY OF HAWAII \\ UNIVERSITY OF TOKYO \\ UNIVERSITY OF UTAH \\ WASHINGTON STATE UNIVERSITY \\ UNIVERSITY OF WASHINGTON \\ AMERICAN MATHEMATICAL SOCIETY
}

The Supporting Institutions listed above contribute to the cost of publication of this Journal, but they are not owners or publishers and have no responsibility for its content or policies.

Mathematical papers intended for publication in the Pacific Journal of Mathematics should be in typed form or offset-reproduced, (not dittoed), double spaced with large margins. Please do not use built up fractions in the text of your manuscript. You may however, use them in the displayed equations. Underline Greek letters in red, German in green, and script in blue. The first paragraph or two must be capable of being used separately as a synopsis of the entire paper. Items of the bibliography should not be cited there unless absolutely necessary, in which case they must be identified by author and Journal, rather than by item number. Manuscripts, in triplicate, may be sent to any one of the editors. Please classify according to the scheme of Math. Reviews, Index to Vol. 39. All other communications should be addressed to the managing editor, or Elaine Barth, University of California, Los Angeles, California, 90024.

The Pacific Journal of Mathematics expects the author's institution to pay page charges, and reserves the right to delay publication for nonpayment of charges in case of financial emergency.

100 reprints are provided free for each article, only if page charges have been substantially paid. Additional copies may be obtained at cost in multiples of 50 .

The Pacific Journal of Mathematics is issued monthly as of January 1966. Regular subscription rate: $\$ 72.00$ a year (6 Vols., 12 issues). Special rate: $\$ 36.00$ a year to individual members of supporting institutions.

Subscriptions, orders for back numbers, and changes of address should be sent to Pacific Journal of Mathematics, 103 Highland Boulevard, Berkeley, California, 94708.

PUBLISHED BY PACIFIC JOURNAL OF MATHEMATICS, A NON-PROFIT CORPORATION

Printed at Kokusai Bunken Insatsusha (International Academic Printing Co., Ltd.), 8-8, 3-chome, Takadanobaba, Shinjuku-ku, Tokyo 160, Japan. 


\section{Pacific Journal of Mathematics}

Patricia Andresen and Marvin David Marcus, Weyl's inequality and

quadratic forms on the Grassmannian .......................

George Bachman and Alan Sultan, Regular lattice measures: mappings and

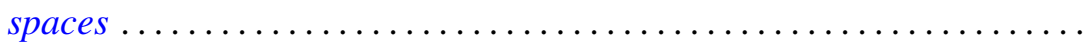

David Geoffrey Cantor, On certain algebraic integers and approximation by rational functions with integral coefficients ...................

James Richard Choike, On the value distribution of functions meromorphic in the unit disk with a spiral asymptotic value ..................

David Earl Dobbs, Divided rings and going-down................ 353

Mark Finkelstein and Robert James Whitley, Integrals of continuous

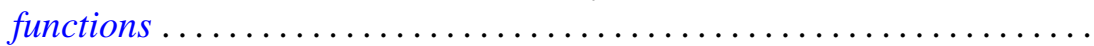

Ronald Owen Fulp and Joe Alton Marlin, Integrals of foliations on manifolds with a generalized symplectic structure ...............

Cheong Seng Hoo, Principal and induced fibrations .................

Wu-Chung Hsiang and Richard W. Sharpe, Parametrized surgery and

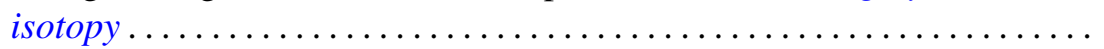

Surender Kumar Jain, Surjeet Singh and Robin Gregory Symonds, Rings whose proper cyclic modules are quasi-injective .................

Pushpa Juneja, On extreme points of the joint numerical range of commuting normal operators...

Athanassios G. Kartsatos, Nth order oscillations with middle terms of order $N-2$.

John Keith Luedeman, The generalized translational hull of a

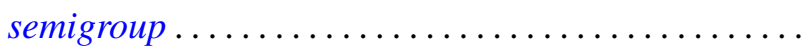

Louis Jackson Ratliff, Jr., The altitude formula and DVR's ...

Ralph Gordon Stanton, C. Sudler and Hugh C. Williams, An upper bound for the period of the simple continued fraction for $\sqrt{D}$...

David Westreich, Global analysis and periodic solutions of second order systems of nonlinear differential equations...

David Lee Armacost, Correction to: "Compactly cogenerated LCA

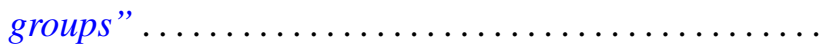

Jerry Malzan, Corrections to: "On groups with a single involution" .

David Westreich, Correction to: "Bifurcation of operator equations with unbounded linearized part" ...................... 